\title{
Quality Adaptation in P2P Video Streaming Based on Objective QoE Metrics
}

\author{
Julius Rückert ${ }^{1}$, Osama Abboud ${ }^{2}$, Thomas Zinner ${ }^{3}$, \\ Ralf Steinmetz ${ }^{2}$, and David Hausheer ${ }^{1}$ \\ 1 P2P Systems Engineering, TU Darmstadt, Germany \\ \{rueckert, hausheer\}@ps.tu-darmstadt.de \\ 2 Multimedia Communications Lab, TU Darmstadt, Germany \\ \{abboud, steinmetz\}@kom.tu-darmstadt.de \\ 3 Chair of Communication Networks, University of Wuerzburg, Germany \\ zinner@informatik.uni-wuerzburg.de
}

\begin{abstract}
The transmission of video data is a major part of traffic on today's Internet. Since the Internet is a highly dynamic environment, quality adaptation is essential in matching user device resources with the streamed video quality. This can be achieved by applying mechanisms that follow the Scalable Video Coding (SVC) standard, which enables scalability of the video quality in multiple dimensions. In SVC-based streaming, adaptation decisions have long been driven by Quality of Service (QoS) metrics, such as throughput. However, these metrics do not well match the way human users perceive video quality. Therefore, in this paper, the classical SVC-based video streaming approach is expanded to consider Quality of Experience (QoE) for adaptation decisions. The video quality is assessed using existing objective techniques with a high correlation to the human perception. The approach is evaluated in context of a P2P-based Video-on-Demand (VoD) system and shows that by making peers favor always layers with a high estimated QoE but not necessarily high bandwidth requirements, the performance of the entire system can be enhanced in terms of playback delay and SVC video quality by up to $20 \%$. At the same time, content providers are able to reduce up to $60 \%$ of their server costs, compared to the classical QoS-based approach.
\end{abstract}

Keywords: Peer-to-Peer Video Streaming, Quality Adaptation, Quality of Experience, Scalable Video Coding.

\section{Introduction}

Recent studies show that the streaming of video content has become a dominating part of today's Internet traffic, with a forecast of further increase for the next years 419. The support of a large number of users as well as a wide range of heterogeneous devices and constantly changing conditions, such as sudden user fluctuations or network congestion, make the provision of appropriate video streaming mechanisms a challenging task. Based on these dynamics, an impact on the provided service quality can sometimes be inevitable. This may become 
visible to the user in form of degraded video quality or stalling during video playback. The goal of content providers is to reduce both effects to a minimum in order to maximize the service quality. This requires quality adaptations that allow for a flexible reaction to provide the individual users with a continuous playback and a maximum possible video quality. In this context, the video codec Scalable Video Coding (SVC) 20] is especially interesting as it allows for flexible adaptation of the video quality in different dimensions. Furthermore, by limiting adaptation decisions to only compatible quality layers, e.g., in terms of bandwidth or screen resolution, heterogeneous clients with different capabilities can be supported in an efficient way.

For quality adaptation during an SVC-based streaming process, the decisions for appropriate layers are essential. Therefore, Quality of Service (QoS) aspects, such as the throughput, are typically taken into consideration to select appropriate quality layers during streaming. However, this approach follows a simplified assumption about video quality and might not directly result in a maximization of the video quality as perceived by a human user. Therefore, the idea proposed in this paper is to extend SVC-based video streaming with the properties of the human visual system in order to judge the influence on the perceived quality, also referred to as Quality of Experience (QoE).

Since the human perception is a complex process and influenced by many factors, only few viable automated metrics, so called objective QoE metrics, exist that can be applied in a general context. At the same time, user studies, which are considered the only reliable alternative to assess perceived quality [15], are not applicable in most technical solutions. Therefore, although automated QoE metrics can only approximate the perception of a user 22, they are a promising approach to enable a more user-centric quality adaptation in SVC based video streaming. In the context of the presented approach, the Video Quality Metric (VQM) [18, a state-of-the-art objective QoE metric, is applied.

To investigate the impact of using objective QoE metrics for quality adaptation, the focus of this paper is on a Peer-to-Peer (P2P) based Video-on-Demand (VoD) scenario. Besides evaluating the adaptation mechanisms on its own, the scenario also allows to study how such adaptations influence the dynamics of a P2P system and the resulting overall performance. For large-scale streaming systems, P2P technologies have proven to be a valid alternative to traditional client-server $(\mathrm{C} / \mathrm{S})$ solutions. They allow to efficiently leverage resources of enduser devices in order to remove bottlenecks and unburden the content provider from high costs. In principle, the adaptation mechanisms presented in this paper could also be applied in a $\mathrm{C} / \mathrm{S}$ scenario, however, they were designed to meet the specific requirements of a highly distributed scenario, with clients autonomously deciding on adaptations according to their needs.

The remainder of this paper is structured as follows. In Sect. 2 an overview about existing work in the field is provided. The QoE-aware adaptation mechanism, which is the main contribution of this paper, is introduced in Sect. 3 . Subsequently, in Sect. 4 the evaluation of the mechanism is presented. Finally, the paper is concluded in Sect. 5 


\section{Related Work}

The topic of quality adaptation in the context of multi-layer and SVC-based video streaming has been broadly studied before. A number of researchers have investigated the general applicability of multi-layer video coding and especially SVC to P2P video streaming 614. Others proposed concrete SVC-based systems to address specialized issues, such as low start-up or streaming delays, or smooth video playback, while trying to assure a minimum video quality [312 16]. In contrast to the approach presented in this paper, none of these works considers QoE characteristics of the content.

In a previous work, the authors evaluated the impact of quality adaptation mechanisms in SVC-based P2P streaming systems [2]. These purely QoS-driven mechanisms were considered and further extended in the context of the presented work to allow for more sophisticated adaptations, also considering QoE aspects.

The QoE during streaming clearly depends on the visual quality of the delivered content. Furthermore, it is also influenced by characteristics of the video streaming process and the quality adaptation mechanism itself. Zinner et al. [26] derived dependencies between SVC quality layers of different videos and their objectively assessed QoE in terms of the Video Quality Metric (VQM) 18. This paper is directly based on this work and uses the results as an input for the presented adaptation mechanism. Lee et al. [11] conducted subjective studies to model the dependencies between SVC quality layers and perceived quality. Others, such as Gustafsson et al. 8], considered more streaming process related issues and evaluated the impact of buffering events on the perceived quality. Zink et al. 25] focused on the role of different layer adaptation characteristics.

Zhai et al. 24] proposed a centralized live SVC video streaming system for wireless, heterogeneous clients that aims at maximizing the QoE. While this work shares the objective of the presented approach to use objectively assessed quality, it greatly differs in its $\mathrm{C} / \mathrm{S}$ scenario and the quality estimation by clients, using own simple objective QoE metrics and local knowledge of the peers. In the context of the presented VoD scenario, it is assumed that content providers could do a pre-processing and this way profit from more sophisticated quality assessments. Furthermore, Kim et al. [10] also proposed a SVC streaming system to maximize the QoE of the users. This approach builds on a centralized dissemination and decision taking approach, where a central entity estimates the perceived quality of a client by using own specialized objective QoE metrics and decides on switching to higher quality layers.

Menkovski et al. 13 described an adaptation mechanism for a distributed streaming system to maintain acceptable video quality. Therefore, direct user feedback and distributed machine learning mechanisms are used to detect unacceptable quality and react by improving the streaming quality. In contrast to the presented work, they do not consider the use of layered video coding but utilize parameters of single layer codecs, including only limited means of adaptability in quality. Furthermore, the approach is limited to two levels of perceived quality which does not allow accounting for heterogeneous clients and fine granular adaption of the video quality. 


\section{QoE-Aware Quality Adaptation}

This section presents how Quality of Experience (QoE) estimation of SVC encoded videos can be used by quality adaptation algorithms to achieve better system performance. Therefore, QoE-aware quality adaptation mechanisms are proposed, designed, and evaluated, that expand the use of classical QoS-driven mechanisms to include other metrics that are closer to how users perceive video quality. What is especially interesting for the performed analysis, is checking whether using estimated QoE in quality layer decisions has any impact on the dynamics of the P2P network and how this influences the performance. For the best of our knowledge, this question has not yet been addressed.

In order to bring QoE to the quality adaptation algorithms in SVC-based VoD systems, an approach is devised that constitutes two major steps: the Quality Management running on a server and the Layer Adaptation running on the peers. The first step is executed by the content provider to derive the QoE ratings for a streamed video. The second step, the actual quality adaptation, takes place in a distributed manner and is autonomously performed by the peers. The quality ratings are disseminated at the beginning of the streaming process and are used by the peers to decide on appropriate video layers during quality adaptations.

\subsection{Quality Management}

The Quality Management step calculates QoE ratings for all SVC layer possibilities of the streamed videos, using an objective QoE metric. This step, depending on the used metric, might be computationally intensive and it is assumed in the considered VoD scenario, that the processing happens offline, executed by the content provider. Currently, a fixed quality rating for a layer over the whole streaming process is assumed, while for longer videos with changing types of content, also multiple rating sets could be used to account for changing characteristics in the quality rating of different scenes.

In the context of this work, the Video Quality Metric (VQM) [18 has been applied to estimate the QoE for the different SVC layers of a video. This objective QoE metric is considered to be state-of-the-art as it was independently evaluated and has proven to correlate with the human perception of video quality for both TV-like video resolutions [7] as well as for high definition videos [23]. Using VQM, deriving the QoE ratings can happen offline without human intervention.

\subsection{Layer Adaptation}

The second step, called Layer Adaptation, extends the QoS-based adaptation mechanisms presented in an earlier work [2. These mechanisms decide on which SVC layers and blocks to request, considering a client's resources and maximizing the throughput. Their decisions are limited by the static resources of the peers and may change over time according to the available real-time resources to allow for a continuous playback. In the presented work, quality decisions take also into consideration the QoE ratings of the Quality Management step, to always 
select the quality layer that does not exceed the peers' bandwidth and has the maximum quality. These ratings depend on a specific video and are distributed to the peers together with other meta data. This could be done by the tracker as the overhead is very small and only transmitted once per video and peer. The rating consists of a single numeric value for each SVC layer.

Quality adaptation in the VoD streaming system is done in two phases. First using the Initial Quality Adaptation (IQA) strategy, an initial quality is selected based on the peer's static resources, for example its bandwidth and screen resolution. After the streaming starts, another set of algorithms called the Progressive Quality Adaptation (PQA) makes sure that streaming and video playback are continuous. If needed, the PQA increases or decreases the video quality when the throughput increases or decreases respectively. Both adaptation phases are extended to maximize the quality according to the QoE ratings.

Since the PQA is executed periodically, there is time between two executions for the adaptation process. Motivated by the fact that too frequent layer variation can have an adverse effect on the quality of experience 25, mechanisms were developed to switch the layer smoothly. Therefore, two steps have been defined for the actual layer adaptation. The first step is the Layer Decision while the second is the Layer Switching. Both take the QoE ratings as input so that to select layers with the highest quality. The layer decision is executed as part of the PQA in order to select a new layer, the so called target layer. The layer switching step follows by defining an adaptation path that starts from the current layer and smoothly changes the quality to the target layer.

Layer Decision. The Layer Decision mechanism decides on a layer that fulfills a certain criterion. In the context of this work, two different strategies for this are considered and compared. Additional strategies are presented in [1].

The first strategy, called $D_{B w}$, has the goal to maximize the bandwidth utilization at the peers and chooses the layer, out of the compatible ones, that has the highest bit rate. This strategy is not QoE-aware as it does not consider any implication on the QoE, rather it focuses on simply fetching the layers with the highest bit rate. This is the state-of-the-art approach for SVC quality adaptation so far and, therefore, used for the sake of comparison and analysis.

The second strategy, called $D_{Q o E}$, constitutes the major contribution of this paper since it uses full knowledge about the QoE ratings of the different SVC layers during quality adaptation. Using the derived QoE rating, as provided by the Quality Management phase, the $D_{Q o E}$ strategy works by selecting the layer that maximizes quality and using it as adaptation target layer.

In Fig. 1 a simple example for $D_{Q o E}$ based on VQM is presented. For the sake of simplicity, the example is 2-dimensional, comprising only spatial and temporal scalability. According to VQM, a QoE rating ranges between 0 (best quality) and 1 (worst quality). The ratings start off with the lowest value for the highest quality at the upper right most block. The values decrease in any dimensions towards the base layer, which has the lowest quality. The selection algorithm iterates over all layers within the illustrated dashed polygon, which 
defines the layers that do not exceed the peer's resources, in terms of, e.g., bandwidth or maximum screen resolution. From these layers, the one with the highest QoE rating and, therefore, the best video quality is chosen.

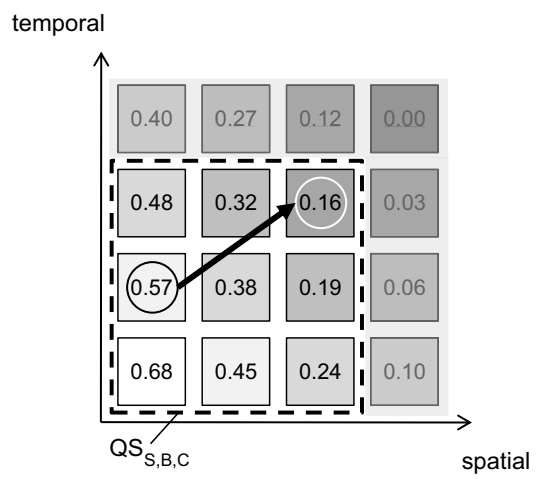

(a) Layer increase

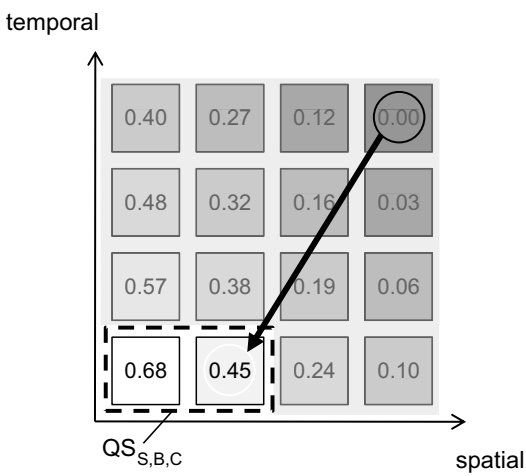

(b) Layer reduction

Fig. 1. Examples for $D_{Q o E}$. Numbers represent the QoE ratings based on VQM.

Layer Switching. Since the time between two adaptation processes can be configured to span several seconds or even minutes, switching between two layers can be done more smoothly by stretching the process over a longer time.

The Layer Switching mechanism defines how to switch to a new layer. In other words, given an old layer $A$ and a new layer $B$ as calculated by the layer decision mechanism, the layer switching algorithms defines the set of layers that have to be passed when switching from $A$ to $B$. The main reason that motivates a smoother switch is the fact that the perceived video quality can be negatively influenced by too frequent quality switches 25 . Therefore, a stepwise adaptation allows to have quality adaptation with smaller steps in between. To avoid having too much layer variation, the mechanism additionally samples the adaptation path in order to put a limit on the amount of steps until the target layer is reached. This process is described at the end of this section.

In the following, the concrete realization of layer switching is presented. The mechanism is referred to as Minimized Absolute Variation in QoE Ratings $\left(S_{Q o E}\right)$. For the sake of clarity, configurations without switching are referred to as Simple Layer Switching $\left(S_{\text {Sim }}\right)$. In this case, switching is done in a simple step by directly jumping to the adaptation's target layer.

The switching mechanism $S_{Q o E}$ uses the variation in QoE ratings of the traversed layers as its minimization metric. Therefore, the goal is to find a path from the old to the new layer which would go through the layers that have the closest QoE ratings. By doing this, the effective quality of the video is changing smoothly between the two layers. In order to implement the switching strategy, graph theory is applied by generalizing the SVC layer model into a graph. Using classical and extensively researched algorithms from graph theory, determines an efficient approach to the problem of minimizing the absolute QoE variation. 
The above described problem is related to the so called Single-Source Shortest Paths problems. Prominent solutions to such problems are the well-known Bellman-Ford and Dijkstra algorithms [5]. The Dijkstra algorithm has been chosen as it exhibits lower complexity and can be easily applied to the presented scenario where the edges between the SVC layers (the variation of QoE ratings) are positive numbers. For the application of the algorithm, in the following the old layer is used as source node while the target layer is used as destination. Before the Dijkstra algorithm can be applied, required for the $S_{Q o E}$ algorithm, first a graph needs to be derived from the SVC layer model. The steps of deriving the graph, applying the Dijkstra algorithm, and interpreting the result as adaptation path are illustrated in Fig. 2.

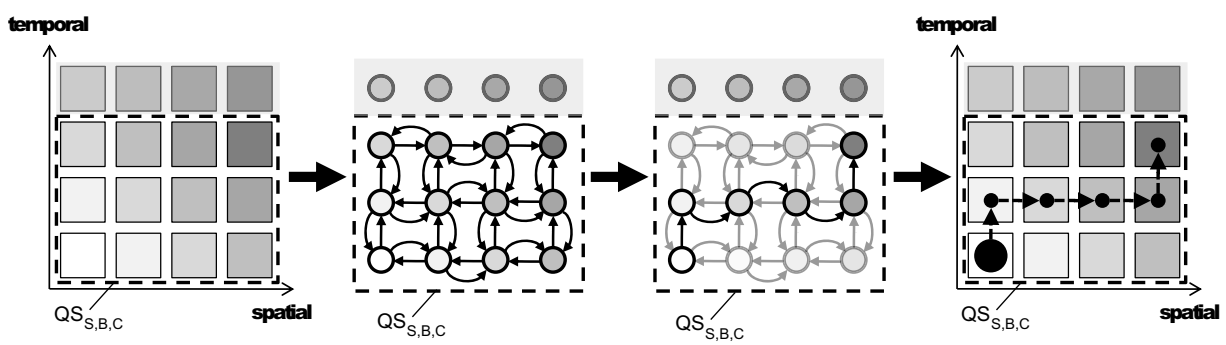

Fig. 2. The steps of interpreting the SVC layer model as graph, retrieving the path with minimal variation from it, and interpretation as adaptation path

To start off, each SVC layer in the layer model is represented as individual node in a graph. Since adaptation is only expected to happen within the set of a peer's compatible layers, in Fig. 2 referred to as $Q S_{S, B, C}$, only the nodes within this set are connected. This way, only supported layers can be reached. Edges are generated by connecting nodes. Since it is considered to switch one layer at a time, each node is only connected to its neighbor nodes that can be reached by adding or removing a single layer in any dimension. Furthermore, the weights of the edges are derived by using the absolute value of the difference between the QoE ratings of the SVC layers presented by the adjacent nodes.

The actual calculation of the switching path is performed by applying the Dijkstra algorithm on the calculated graph. The Dijkstra algorithm ensures that the retrieved path minimizes the switching variation of the QoE ratings.

Sampling the Retrieved Paths. Since the main objective of performing Layer Switching is to have smooth transitions, it is essential not to overwhelm the user with too many layer variations 25. Therefore, it might not be beneficial to switch through all layers along a derived switching path. Thus, a sampling of the derived adaptation path has been introduced to limit the number of jumps in a given time interval. Motivated by $\mathrm{Ni}$ et al. [17, a minimal time of 3 seconds has been chosen per switch. Given a default adaptation interval of 10 seconds, this allows for three steps per adaptation. 
Configuration of Strategies. For the sake of clarity, an adaptation strategy is referred to by the name $D_{x} S_{y}$, where $x$ denotes the used Layer Decision and $y$ the Layer Switching algorithm. This way, $D_{B w} S_{S i m}$, for example, refers to the mechanisms that combine Layer Decision maximizing bandwidth utilization and the simple Layer Switching algorithm.

\section{Evaluation Results}

A VoD scenario was considered that comprises nine streaming servers and 100 peers with three different resource classes to reflect peer heterogeneity. The used number of peers has been shown to be typical in content distribution scenarios 9. The peers differ in their static device properties such as the maximum screen resolution and connection bandwidth. The VoD system uses a mesh-/pullbased dissemination scheme. For an accurate simulation of the transferred video streams, all properties of the videos were derived from real SVC encoded videos. The length of the test video is 300 seconds, which is a typical duration of, e.g., a video shared at YouTube. Peers enter the system with an exponentially distributed arrival time with a mean of 90 seconds. They stay in the system after finishing the streaming process with a probability of $40 \%$ for a random time between 0 and 300 seconds. During streaming, progressive quality adaptation takes place every 10 seconds, which has been shown in a previous work to be a reasonable value [2]. All simulations were conducted using the simulation framework PeerfactSim.KOM [21]. They were repeated 10 times with different seeds for each configuration, 95-percent confidence intervals are reported for all results. Further details on the simulation parameters and the setup as well as additional scenarios are described in [1].

Evaluation Metrics. For the evaluation, two classes of metrics were used: session metrics, describing characteristics of the streaming process, and SVC video quality metrics focusing on the quality of the delivered video content.

The class of session metrics includes the start-up delay, the total stalling time, the number of stalling events, the number of SVC layer changes, and the SVC layer change amplitude. The start-up delay is the time between starting the streaming process and the time until enough initial data were received to start the playback. The total stalling time is defined by the sum of all stalling event durations over the playback process, including the start-up delay. The number of stalling events describes how often the playback was paused due to missing data. The number of SVC layer changes and the change amplitude describe how often quality layer changes happened and how far jumps between two layers were. The SVC layer change amplitude is defined as follows:

$$
\text { Amplitude }\left(l_{1}, l_{2}\right)=\left|d_{1}-d_{2}\right|+\left|t_{1}-t_{2}\right|+\left|q_{1}-q_{2}\right|,
$$

where $l_{1}=\left(d_{1}, t_{1}, q_{1}\right)$ and $l_{2}=\left(d_{2}, t_{2}, q_{2}\right)$ describe the two involved SVC layers as triple of their spatial (s), temporal (t), and quality (q) layer dimensions. 
For the class of SVC video quality metrics, the relative received layer and the VQM rating were used. The relative received layer describes the ratio of a peer's received SVC layer to its requested maximum layer. A value of 1 denotes that a peer received the video with the maximum requested quality. The VQM rating describes the estimated QoE for the received SVC layers, derived using the objective QoE metric VQM [18]. For this metric a lower value denotes a better quality rating. For clarity, the mapping to the Mean Opinion Score (MOS) is included in the evaluation figures as described by Zinner et al. [26].

System Capacity. In a first step, the impact of changing server resources on the system performance and the streaming process were assessed. Good provisioning of server capacity is essential for content providers who want to provide good performance but still keep costs low. For the experiments, the upload bandwidth of the streaming servers was used as target parameter. In Fig. 3, a subset of the results are presented, including the start-up delay, total stalling time, relative received quality, and played-out VQM rating.

The following observations can be made for the session quality: Increasing the server capacity yields almost an exponential decrease in the start-up delay for both mechanisms. The QoE-aware mechanism $D_{Q o E} S_{Q o E}$ shows clear advantage (of one second) over the QoS-based $D_{B w} S_{\text {Sim }}$ mechanism. Starting from $25 \mathrm{Mbps}$ server capacity, a saturation effect can be observed, where adding more capacity does not further decrease the start-up delay. This can be attributed to the time the buffer has to be filled, which is limited by the download speed of the peers and the server capacity. A similar observation can be made for the total stalling time, where both mechanisms show a saturation effect for capacities above $25 \mathrm{Mbps}$, with the QoE-aware mechanism constantly having a slightly better performance.

For the SVC video quality the following observation can be made: First of all, increasing server capacity enables the peers to better maintain the initially selected layer and this way achieve high relative received quality values. When a content provider is offering a high server upload capacity of $55 \mathrm{Mbps}$, the relative received quality reaches $80 \%$ and $90 \%$ for the $D_{B w} S_{S i m}$ and the $D_{Q o E} S_{Q o E}$ mechanisms respectively. For the QoE-aware adaptation mechanism this is already achieved starting from $25 \mathrm{Mbps}$. For content providers it is possible to save precious server resources using the proposed QoE-aware mechanism. Assuming, for example, a target average received layer of $80 \%$, for $D_{B w} S_{\text {Sim }}$ this would require server resources of $35 \mathrm{Mbps}$, whereas for $D_{Q o E} S_{S i m} 15 \mathrm{Mbps}$ would be sufficient, resulting in up to $60 \%$ reduced server resources. This can be explained by the fact that since the peers are favoring layers with higher VQM ratings, those layers are better replicated, making it easier to retrieve video data from other peers rather than from the server. For the VQM rating, it can be observed that, starting from a $15 \mathrm{Mbps}$ server capacity, the $D_{Q o E} S_{Q o E}$ mechanisms yields an average played VQM quality of 0.2 . This maps to an excellent perceived quality on the MOS scale. $D_{B w} S_{\text {Sim }}$ only achieves a good level of quality.

To sum these results up, it can be concluded that using the QoE-aware mechanism a great reduction of server capacities by up to $60 \%$ is possible in comparison to the used non-QoE-aware mechanism, while providing a high level of 


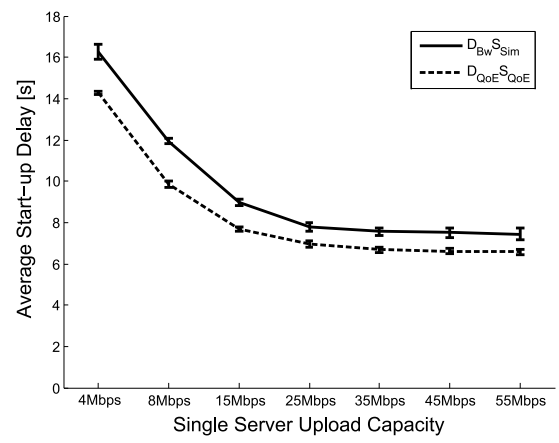

(a) Average start-up delay per peer

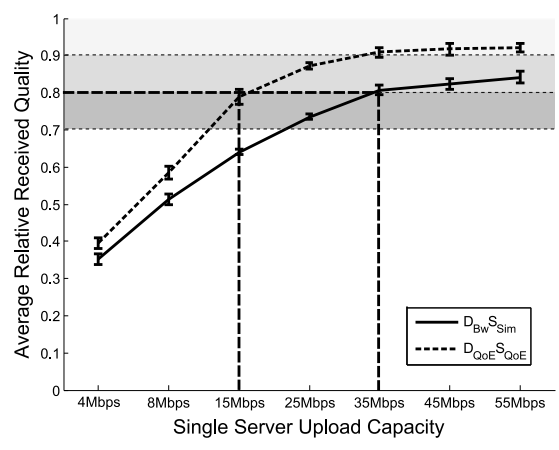

(c) Average relative received quality

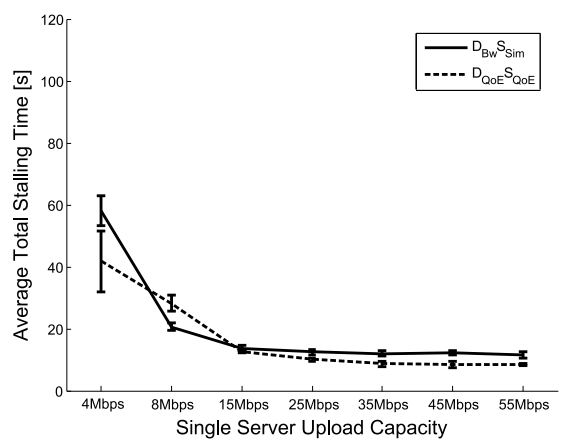

(b) Average total stalling time per peer

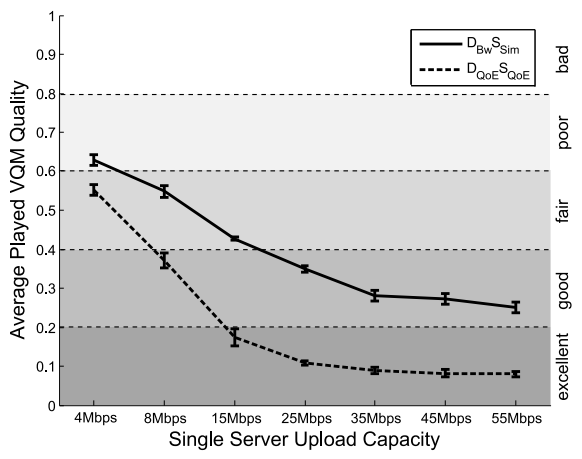

(d) Average played VQM quality

Fig. 3. Comparison of session and video quality for changing system capacities

relative received quality of $80 \%$ and improving the VQM to the highest quality level on the MOS scale. Using these results, enables content providers to define a threshold for the relative received quality, or even better the estimated perceived quality in terms of the VQM rating, and provide additional server capacities on demand, if a certain critical limit is about to be violated.

Adaptation Strategies. In a second step, the impact of using the proposed QoE-aware mechanism with and without Layer Switching was evaluated. As described before, the concept of Layer Switching was introduced to allow for a smooth change in layers by using an adaptation path and a stepwise execution. For the evaluation, again the default configuration was used, this time with a fixed maximum upload bandwidth for each server of $25 \mathrm{Mbps}$.

In Fig. 4, the observed impact of the different adaptation mechanisms on the session quality is shown. As already observed before, using the QoE-aware mechanisms, the total stalling time of the peers can be reduced. While for the non-QoE-ware mechanism $D_{B w} S_{S i m}$ an average value of about 12 seconds is observable, $D_{Q o E} S_{S i m}$ and $D_{Q o E} S_{Q o E}$ allow for a reduction of more than 2 seconds. The high degree of improvement becomes further apparent in the second 
used session metric, the number of stalling events. Here reductions of observed stalling events over the whole simulation from values of about 110 for $D_{B w} S_{S i m}$, to about 40 for $D_{Q o E} S_{S i m}$, and about 50 for $D_{Q o E} S_{Q o E}$ could be shown. For $D_{Q o E} S_{S i m}$ and $D_{Q o E} S_{Q o E}$, this relates to a decrease of more than $50 \%$.

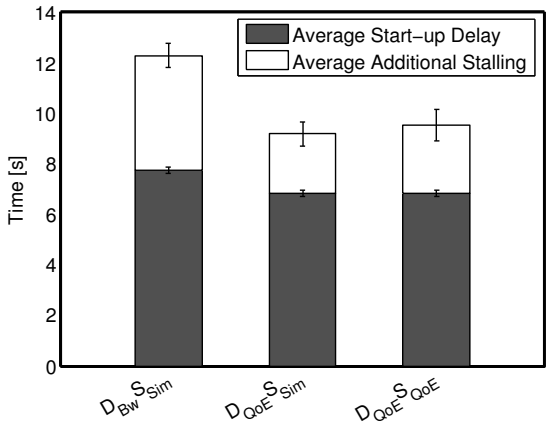

(a) Total stalling time per peer

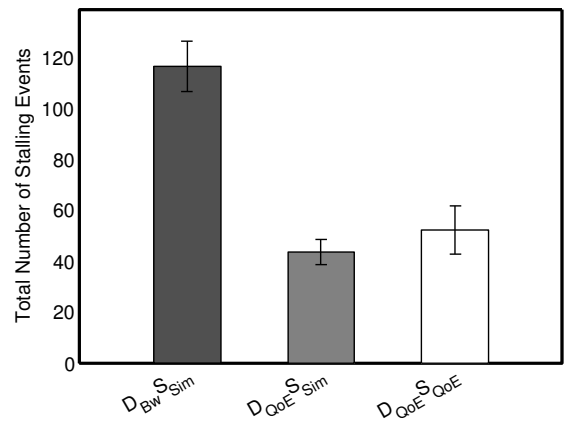

(b) Overall number of stalling events

Fig. 4. Comparison of the session quality for different adaptation mechanisms

In Fig. 5 the impact on two further session metrics is shown. For the number of layer changes due to adaptations, a reduction of more than $50 \%$ from the non-QoE to the simple QoE-aware mechanisms is observable. The introduction of Layer Switching, in contrast, led again to a slight rise. This and the results presented before imply that using Layer Switching leads to a reduction of the session quality. In order to, nevertheless, show the impact of this mechanism, the layer change amplitude has been introduced as session metric. The motivation for this was the observation that not only the number of changes but also the step width of changes has an important impact on the perceived quality and should be kept as small as possible [25]. The metric was defined at the beginning of the section. In Fig. 5b, the result for the layer change amplitude is presented. Compared to the $D_{B w} S_{\text {Sim }}$ mechanism, an increase for this metric can be observed using the simple QoE-aware mechanism. Additionally, using Layer Switching allows to reduce the amplitude to a value even below the value of the non-QoE-aware mechanism. Layer Switching, therefore, may further help to improve the perceived quality.

To understand the relevance of the different adaptation mechanisms for the QoE, the average received VQM rating for the delivered content is presented in Fig. 6. The two most important observations are that, using the QoE-aware mechanisms, the objective QoE rating can be improved from a good to an excellent level and that, in contrast to the non-QoE-aware mechanism, peers with more resources are able to retrieve content with a better quality rating. For the non-QoE-aware mechanisms the opposite was the case. 


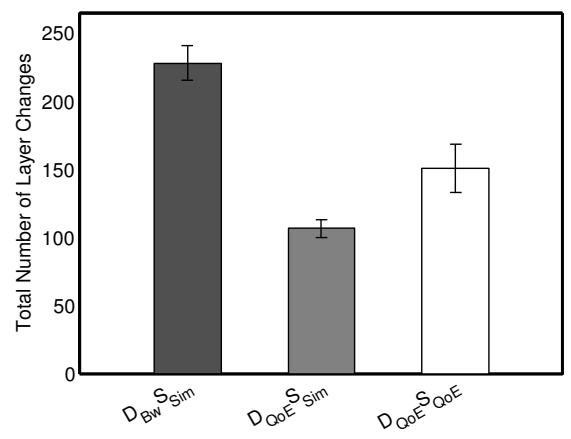

(a) Overall number of layer changes

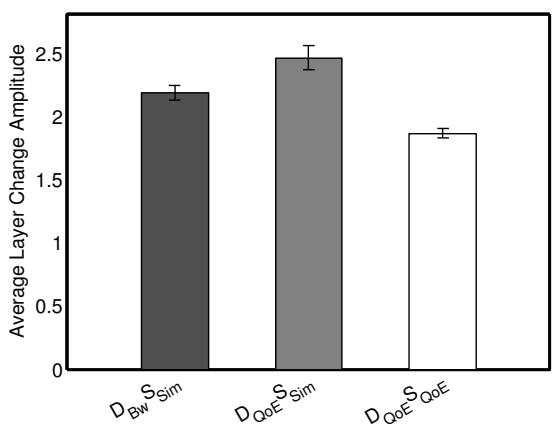

(b) Average Layer Change Amplitude

Fig. 5. Layer change characteristics for different adaptation mechanisms

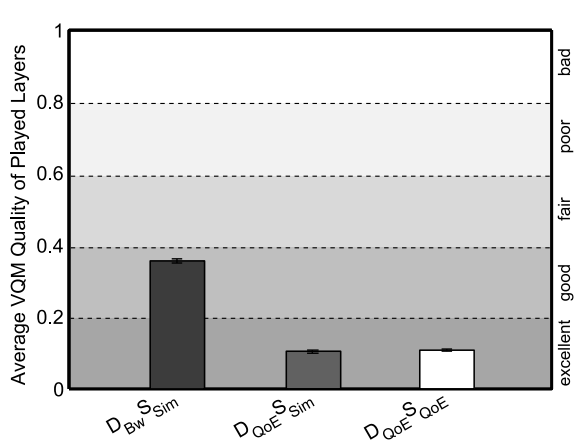

(a) Played VQM quality

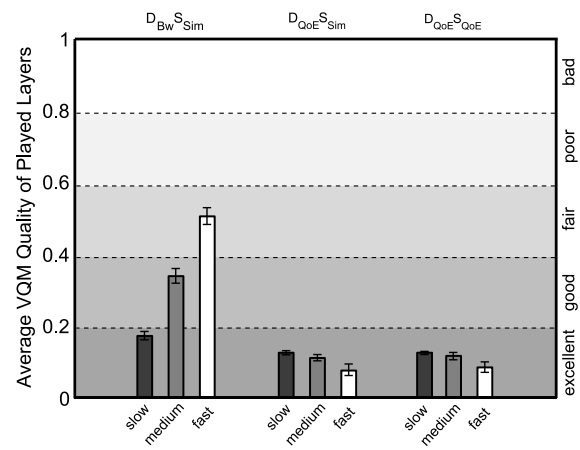

(b) Played VQM quality per peer group

Fig. 6. Comparison of the VQM quality for different adaptation mechanisms

\section{Conclusion and Future Work}

This paper investigated how objective assessment of perceived video quality can be used to realize quality adaptation mechanisms for SVC-based video streaming. Therefore, the design of a QoE-aware quality adaptation mechanism using the state-of-the-art objective QoE metric VQM has been presented. Furthermore, the approach was evaluated in the context of a P2P VoD streaming scenario.

The evaluation results show that by applying the proposed mechanisms, a content provider could benefit from lower costs due to a decrease in required streaming server resources by up to $60 \%$, compared to traditional QoS-driven mechanisms. The QoE-aware adaptation mechanism showed superior advantages over the non-QoE-aware mechanisms in terms of lower total stalling event counts by up to $50 \%$, as well as the relative received quality of the single peers by up to $20 \%$. Besides, it could be shown that the proposed mechanism succeeds in maximizing the objectively estimated perceived quality, which is the target metric of the mechanism. By additionally using Layer Switching mechanisms 
for smooth transitions during layer changes, the average layer change amplitude can be reduced, at the cost of an increased total number of layer changes. For the latter result it is assumed that subjective studies have to be conducted to further evaluate the two parameters' impact on the perceived quality by human users. The results then can be applied to decide on how these parameters have to be weighted to maximize the QoE during the streaming process.

As future work, the consideration of longer videos is planned. Therefore, it has to be investigated how one can account for changes in the video content, as, e.g., implied by different scenes in a movie. As the perception of video quality is highly dependent on the content, it is assumed that different quality ratings are necessary for different parts of a longer video. Additionally, a more detailed analysis of the adaptation strategies' impact on the perceived quality is planned, including the consideration of the QoE for single users. In this context, it is of particular interest how the strategies affect the users with the worst QoE in the system. Finally, it is planned to perform a prototypical evaluation of the presented adaptation mechanisms in a testbed to investigate their applicability in real-world environments.

Acknowledgements. This work has been supported in parts by the IT R\&D program of MKE/KEIT of South Korea (10035587, Development of Social TV Service Enabler based on Next Generation IPTV Infrastructure), by the Federal Ministry of Education and Research of the Federal Republic of Germany (support code 01BK0806, G-Lab), and by the German Research Foundation (Research Group 733, QuaP2P: Quality Improvement of Peer-to-Peer Systems).

\section{References}

1. Abboud, O., Rückert, J., Hausheer, D.: QoE-aware Quality Adaptation in Peerto-Peer Video-on-Demand. Tech. Rep. PS-TR-2012-01, Peer-to-Peer Systems Engineering, TU Darmstadt (2012)

2. Abboud, O., Zinner, T., Pussep, K., Steinmetz, R.: On the Impact of Quality Adaptation in SVC-based P2P Video-on-Demand Systems. In: ACM MMSys (2011)

3. Baccichet, P., Schierl, T., Wiegand, T., Girod, B.: Low-delay Peer-to-Peer Streaming using Scalable Video Coding. In: IEEE PV Workshop (2007)

4. Cisco Systems Inc.: Cisco VNI: Forecast and Methodology, 2010-2015 (2011), http://www.cisco.com/en/US/solutions/collateral/ns341/ns525/ns537/ ns705/ns827/white_paper_c11-481360.pdf (accessed November 12, 2011)

5. Cormen, T.H., Leiserson, C.E., Rivest, R.L., Stein, C. (eds.): Introduction to Algorithms, 2nd edn. The MIT Press (2001)

6. Cui, Y., Nahrstedt, K.: Layered Peer-to-Peer Streaming. In: ACM NOSSDAV (2003)

7. Video Quality Experts Group: Final Report on the Validation of Objective Models of Video Quality Assessment, FR-TV Phase II (2003), http://www.its.bldrdoc.gov/vqeg/project-pages/frtv-phase-ii/ frtv-phase-ii.aspx (accessed November 12, 2011)

8. Gustafsson, J., Heikkila, G., Pettersson, M.: Measuring Multimedia Quality in Mobile Networks with an Objective Parametric Model. In: IEEE ICIP (2008) 
9. Kaune, S., Tyson, G., Pussep, K., Mauthe, A., Steinmetz, R.: The Seeder Promotion Problem: Measurements, Analysis and Solution Space. In: IEEE ICCCN (2010)

10. Kim, C.S., Sohn, H., Neve, W.D., Ro, Y.M.: An Objective Perceptual QualityBased ADTE for Adapting Mobile SVC Video Content. IEICE Trans. on Information \& Systems E92-D(1), 93-96 (2009)

11. Lee, J., Simone, F.D., Ebrahimi, T.: Subjective Quality Evaluation via Paired Comparison: Application to Scalable Video Coding. IEEE Trans. on Multimedia 13(5), 882-893 (2011)

12. Lee, T.C., Liu, P.C., Shyu, W.L., Wu, C.Y.: Live Video Streaming Using P2P and SVC. In: IFIP/IEEE MMNS (2008)

13. Menkovski, V., Exarchakos, G., Liotta, A.: Machine Learning Approach for Quality of Experience Aware Networks. In: IEEE INCoS (2010)

14. Mokhtarian, K., Hefeeda, M.: Analysis of peer-assisted Video-on-Demand Systems with Scalable Video Streams. In: ACM MMSys (2010)

15. Mu, M.: An Interview with Video Quality Experts. ACM SIGMultimedia Records 1(4), 4-13 (2009)

16. Mushtaq, M., Ahmed, T.: Smooth Video Delivery for SVC based Media Streaming over P2P Networks. In: IEEE CCNC (2008)

17. Ni, P., Eg, R., Eichhorn, A., Griwodz, C., Halvorsen, P.: Flicker Effects in Adaptive Video Streaming to Handheld Devices. ACM Multimedia (2011)

18. Pinson, M., Wolf, S.: A New Standardized Method for Objectively Measuring Video Quality. IEEE Trans. on Broadcasting 50(3), 312-322 (2004)

19. Sandvine Inc.: Fall 2011 Internet Phenomena Report (2011), http://www.sandvine.com/downloads/documents/10-26-2011_phenomena/ Sandvine $\% 20$ Global $\% 20$ Internet $\% 20$ Phenomena $\% 20$ Report $\% 20-\% 20 \mathrm{Fall} \% 202011$.pdf (accessed November 12, 2011)

20. Schwarz, H., Marpe, D., Wiegand, T.: Overview of the Scalable Video Coding Extension of the H.264/AVC Standard. IEEE TCSVT 17(9), 1103-1120 (2007)

21. Stingl, D., Groß, C., Rückert, J., Nobach, L., Kovacevic, A., Steinmetz, R.: PeerfactSim.KOM: A Simulation Framework for Peer-to-Peer Systems. In: ACM/IEEE/IFIP HPCS (2011)

22. Winkler, S.: Digital Video Quality: Vision Models and Metrics, 1st edn. Wiley (2005)

23. Wolf, S., Pinson, M.: Application of the NTIA General Video Quality Metric (VQM) to HDTV Quality Monitoring. In: VPQM (2007)

24. Zhai, G., Cai, J., Lin, W., Yang, X., Zhang, W.: Three Dimensional Scalable Video Adaptation via User-End Perceptual Quality Assessment. IEEE Trans. on Broadcasting 54(3), 719-727 (2008)

25. Zink, M., Künzel, O., Schmitt, J., Steinmetz, R.: Subjective Impression of Variations in Layer Encoded Videos. In: IEEE IWQoS (2003)

26. Zinner, T., Hohlfeld, O., Abboud, O., Hoßfeld, T.: Impact of Frame Rate and Resolution on Objective QoE Metrics. In: IEEE QoMEX Workshop (2010) 